\title{
Riesgo operacional en el proceso de pago del Procampo Un enfoque bayesiano
}

Fecha de recepción: 29.11.2011

Fecha de aceptación: 16.01.2012

José Francisco

Martínez Sánchez

Escuela Superior de Economía, Instituto Politécnico Nacional marzan67@hotmail.com

\section{Francisco}

Venegas Martínez

Escuela Superior de Economía, Instituto Politécnico Nacional fvenegas1111@yahoo.com.mx

\section{Resumen}

Este trabajo identifica y cuantifica, a través de un modelo de red bayesiana (RB), los diversos factores de riesgo operacional (RO) asociados al proceso de pago del Programa de Apoyos Directos al Campo (Procampo). El modelo de RB es calibrado con datos de eventos que se presentaron durante el periodo 2008-2011. A diferencia de los métodos clásicos, la calibración del modelo de RB incluye fuentes de información tanto objetivas como subjetivas, lo cual permite capturar de manera más adecuada la interrelación (causa-efecto) entre los diferentes factores de riesgo operativo.

Palabras clave: riesgo operacional, análisis bayesiano, simulación Monte Carlo.

Clasificación JEL: D81, C11, C15. 


\title{
Operational risk in the payment process of Procampo. A bayesian approach
}

\begin{abstract}
This paper identifies and quantifies through a Bayesian Network model (BN) the various factors of Operational Risk (OR) associated with the payment process PROCAMPO. The BN model is calibrated with data from events that occurred during the period 2008-2011. Unlike classical methods, the BN model calibration sources include both objective and subjective ones, allowing to more adequately capture the relationship (cause and effect) between the several elements of operational risk.
\end{abstract}

Keywords: operational risk, bayesian analysis, Monte Carlo simulation

JEL Clasification: D81, C11, C15.

\section{Introducción}

El enfoque bayesiano es una alternativa viable para el análisis de riesgos en condiciones de incertidumbre. Por construcción, los modelos bayesianos incorporan información inicial a través de una distribución de probabilidad a priori, mediante la cual se puede incluir información subjetiva en la toma de decisiones como la opinión de expertos, el juicio de analistas o las creencias de especialistas. Este trabajo utiliza un modelo de red bayesiana (RB) para examinar la interrelación entre factores de riesgo operacional ${ }^{1}(\mathrm{RO})$ en el proceso de pago del Programa de Apoyos Directos al Campo (Procampo). El modelo RB que se propone se calibra con datos observados en eventos que se presentaron durante el proceso de bancarización ${ }^{2}$ y con información que se obtuvo de los expertos ${ }^{3}$ o externa; el periodo de análisis es el comprendido desde 2008 hasta 2011.

A pesar de la existencia de trabajos — como los de Reimer y Neu $(2002,2003)$, Kartik y Reimer (2003), Leippold (2003), Aquaro et al. (2009), Neil, Márquez y Fenton (2004) y Alexander (2002) — que abordan de manera general la aplicación

\footnotetext{
${ }^{1}$ También conocido como riesgo operativo.

${ }^{2}$ La bancarización es el cambio de medio de pago de cheque a transferencia electrónica.

${ }^{3}$ Cuando se haga referencia a expertos nos referimos a funcionarios de la Secretaría de Agricultura, Ganadería, Desarrollo Rural, Pesca y Alimentación (Sagarpa) que tienen la experiencia y conocimiento sobre la operación y administración del proceso de pago de apoyos directos de Procampo.
} 
de las RB en la administración del RO, no existe una guía completa sobre cómo clasificar los eventos de RO, cómo identificarlos, cómo cuantificarlos y cómo calcular el capital económico de manera consistente. ${ }^{4}$ Este trabajo pretende cerrar estas brechas de la siguiente forma: primero, establecer estructuras de información sobre eventos de RO de manera que sea posible identificar, cuantificar y medir el RO; posteriormente, cambiar el supuesto de independencia de eventos para modelar de manera realista el comportamiento causal de los eventos de RO.

La posibilidad de utilizar funciones de distribución condicionales, discretas o continuas, calibrar el modelo con fuentes de información, tanto objetivas como subjetivas, y establecer una relación causal entre los factores de riesgo, son los rasgos que precisamente distinguen esta investigación en comparación con los modelos estadísticos clásicos.

Este trabajo se organiza de la siguiente forma. En primer lugar, se presenta la terminología que se empleará para el cálculo de RO de acuerdo con los procesos y sistemas de pago relacionados con el Procampo. Después, se analiza el marco teórico para el desarrollo del trabajo, enfatizando sobre las características y bondades de las RB. Posteriormente, se describe la problemática que se pretende resolver, así como el alcance de la aplicación de la metodología propuesta. En seguida, se construyen dos redes: una para la frecuencia y otra para la severidad; para cuantificar cada nodo de las redes y obtener las probabilidades a priori se "ajustan" distribuciones de probabilidad para los casos donde existe información histórica; en caso contrario se recurre a la opinión o juicio de los expertos para obtener las probabilidades correspondientes; una vez que se cuenta con las probabilidades $a$ priori de las dos redes, se procede a calcular las probabilidades a posteriori a través de algoritmos de inferencia bayesiana, en específico se utiliza el algoritmo junction tree. ${ }^{5}$ A continuación, se calcula el riesgo operacional condicional a través de simulación Monte Carlo con las distribuciones a posteriori calculadas para la frecuencia y la severidad; asimismo, se calcula la máxima pérdida esperada con el modelo clásico y se comparan ambos resultados. Por último, se presentan las principales conclusiones de este trabajo.

\footnotetext{
${ }^{4}$ Usualmente, para medir la máxima pérdida esperada (o capital económico) por RO se utiliza el Valor en Riesgo Condicional (CVaR).

${ }^{5}$ Ver Apéndice A.
} 


\section{Sistema de pagos}

\section{Antecedentes}

El sistema de pagos de Procampo fue desarrollado gradualmente por Apoyos y Servicios a la Comercialización Agropecuaria (Aserca) a partir de 1993 para hacer llegar directamente a los productores los recursos federales derivados de la operación de Procampo, operado por Aserca. ${ }^{6} \mathrm{Al}$ integrar el padrón de beneficiarios del citado Programa con más de 2.7 millones de productores y con una superficie apoyada de, aproximadamente, 14 millones de hectáreas cultivables — distribuida en 4.2 millones de predios- se creó la base de datos más completa del sector. Así, pues, Procampo y el sistema de pagos que lo acompaña tienen el mismo origen temporal y la misma explicación causal; pero, en forma adicional y progresiva, este sistema y su amplia base de datos fueron aprovechados para la emisión y distribución de apoyos de otros programas de la Secretaría como Programa de Estímulos a la Productividad Ganadera (Progan), apoyos al café, comercialización, rastros TIF (Tipo Inspección Federal), energéticos agropecuarios, entre otros.

\section{Estructura del sistema de pagos}

El sistema de pagos es el instrumento que tiene la Secretaría de Agricultura, Ganadería, Desarrollo Rural, Pesca y Alimentación (Sagarpa) para administrar el pago de subsidios de los diferentes programas; actualmente operado por Aserca, está estructurado en tres subsistemas: gobernabilidad, procesos sustantivos y tecnología informática.

Desde el punto de vista de la gobernabilidad, el pago de subsidios se apoya en el sistema de pagos para el cumplimiento de los objetivos y líneas de acción establecidos en el Programa Sectorial de Desarrollo Agropecuario y Pesquero, dentro de las cuales se destaca:

I. Poner en marcha los nuevos esquemas de Procampo y Progan (Programa de Producción Pecuaria Sustentable y Ordenamiento Ganadero y Apícola) con nuevas reglas de operación.

II. Establecer un sistema bancarizado.

${ }^{6}$ Órgano desconcentrado de la Sagarpa. 
Respecto al proceso sustantivo del sistema de pagos, éste involucra cuatro subprocesos a través de los cuales recibe, procesa, calcula y emite, anualmente, alrededor de 5.8 millones de solicitudes de pago de apoyos. El 84\% corresponde a Procampo; el $1.57 \%$, a comercialización; el $8.91 \%$, a energéticos; y el $5.5 \%$, a otros programas (fomento café, pro-oleaginosas, cítricos).

En cuanto a la tecnología informática del sistema de pagos, actualmente está conformada por una infraestructura con los siguientes componentes:

I. Desarrollo de aplicaciones (lenguajes de programación y equipos)

II. Administración de bases de datos (bases de datos y servidores)

III.Mesa de ayuda y explotación de información (sistema de control de incidencias)

IV. Soporte técnico (red de voz y datos, equipos y periféricos, correo electrónico y directorio activo)

V. Servicios informáticos (telecomunicaciones, alojamiento, mantenimiento y licenciamiento de bases de datos y servidores de cómputo)

VI. Interfaces con el sistema bancario y financiero

\section{Bancarización}

En los últimos tres años se ha logrado un avance muy significativo en sustituir los cheques, como medio de pago, por transferencias electrónicas a las cuentas de los beneficiarios que se han abierto para tal efecto a través de convenios con las instituciones financieras participantes; este proceso se conoce como bancarización. En el ejercicio fiscal de 2010, el $31 \%$ de las transferencias a los beneficiarios de Procampo se realizó a través de cheques y el $60 \%$ a través de transferencias o depósitos a las cuentas de los beneficiarios. Para 2011 la participación de las transferencias será mucho mayor, toda vez que al cierre de 2010 se habían abierto cuentas a 2168000 beneficiarios, de los cuales sólo a 1552706 se les pagó el apoyo con depósito en ese año. 


\section{Cuadro 1}

Distribución de pagos por medio de pago

\begin{tabular}{c|c|c}
\hline \multicolumn{2}{|c}{$\begin{array}{r}\text { Transferencias a los beneficiarios de Procampo por medio } \\
\text { de pago. Ejercicio fiscal 2010 }\end{array}$} \\
\hline Medio de pago & Cantidad & $\begin{array}{c}\text { Estructura } \\
\text { porcentual }\end{array}$ \\
\hline Cheques & 1133913 & 31.06 \\
\hline $\begin{array}{c}\text { Depósitos banca } \\
\text { comercial }\end{array}$ & 2191555 & 60.04 \\
\hline Depósitos Tesofe & 324941 & 8.90 \\
\hline Total & 3650409 & 100.00 \\
\hline
\end{tabular}

Fuente: Aserca

\section{Ventajas de la bancarización}

La bancarización erradica prácticas no deseables que se han detectado en el proceso de entrega de los cheques a los beneficiarios; muchas veces aquéllos son endosados por el beneficiario a una tercera persona - ya sea por su voluntad o mediante engaños - que paga en efectivo al beneficiario una cantidad menor al importe nominal del cheque. En ciertos casos esta práctica se puede considerar un servicio que se brinda al beneficiario al darle liquidez a partir de los cheques. Estos instrumentos son líquidos si y sólo si existe una infraestructura bancaria adecuada dentro de un rango de distancia razonable. En otros casos, las prácticas de cambio de cheques por dinero en efectivo son abusos hacia los beneficiarios por parte de los prestadores de distintos servicios (tenderos, restauranteros, etc.); agentes diversos de la sociedad rural (iglesias, autoridades ejidales, organizaciones económicas, etc.); o bien, son prácticas de corrupción cuando involucran a funcionarios públicos. Estas prácticas reflejan muchas veces la existencia de factores de poder en la sociedad rural. Algunas de ellas podrían replicarse bajo el nuevo esquema de pagos mediante transferencias, a través de exacciones, chantajes y presiones a los beneficiarios.

Con la bancarización los beneficiarios pueden acceder a sus recursos en efectivo sin recurrir a ningún tipo de intermediarios al acudir a los cajeros automáticos o sucursales bancarias. Sólo en casos extremos en los que los beneficiarios no pueden hacer sus retiros por cuestiones de edad, educación, o rechazo cultural, recurren a un familiar o amigo para que les auxilie en el retiro del dinero. 


\section{Identificación de riesgos en la bancarización}

Para identificar los factores de riesgo asociados a la bancarización se utilizó la técnica denominada análisis causa-efecto (Ishikawa, 1943). Al analizar los riesgos y sus factores, estaremos identificando fallas en los procesos, duplicidad de actividades, actividades que no agregan valor, fallas en los servicios de terceros, entre otros.

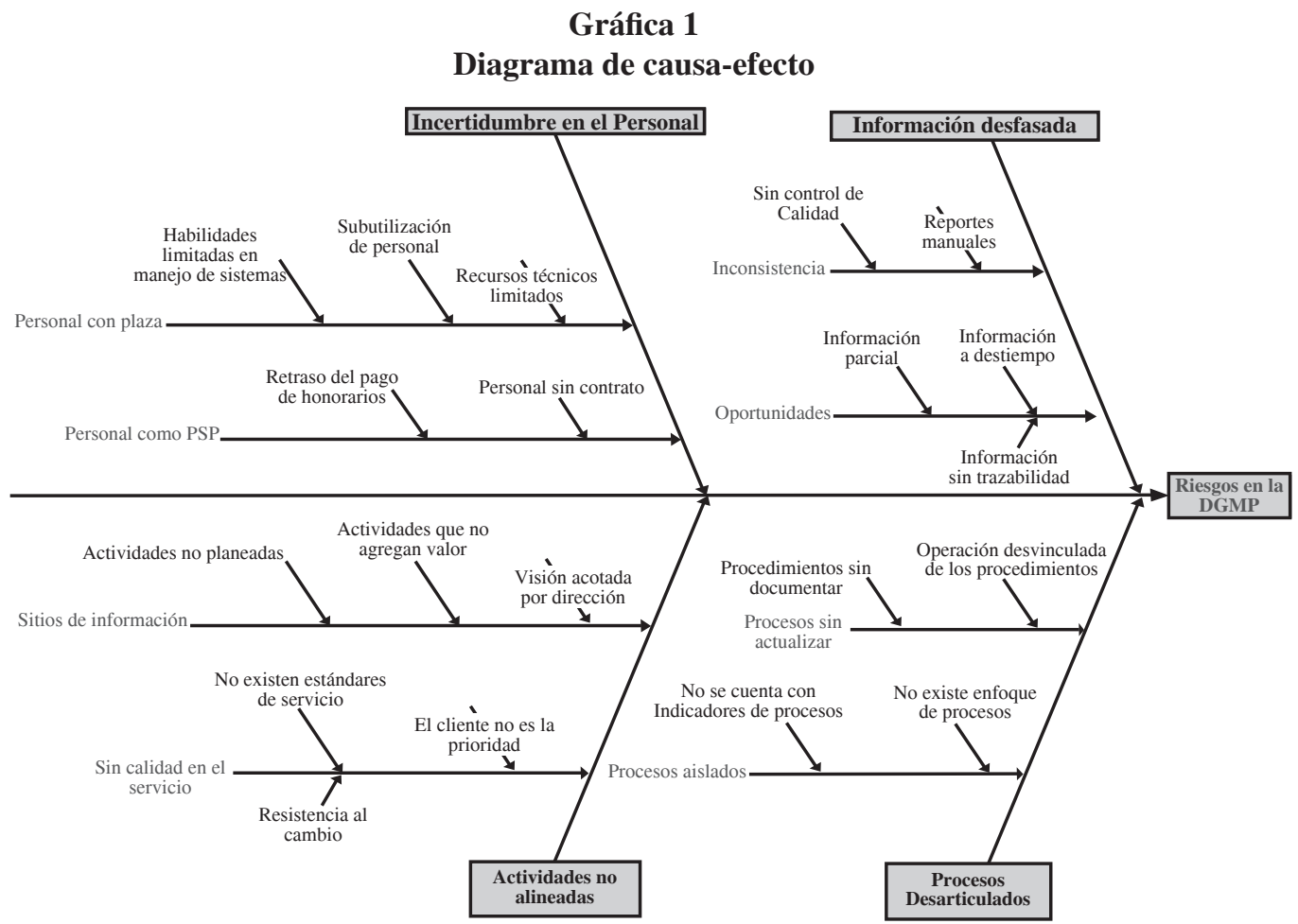

Con base en el análisis anterior se identificaron los nodos (variables aleatorias) que formarán parte de la RB, las cuales se describen y analizan estadísticamente más adelante.

\section{Medición del riesgo operacional}

La naturaleza de los métodos para la cuantificación y medición del riesgo operacional varía desde lo más simple hasta métodos de gran complejidad y entre modelos que consideran un solo indicador y modelos estadísticos muy sofisticados. 


\section{Métodos para medir el riesgo operacional}

A continuación se describen los métodos existentes en la literatura para medir el RO (véanse, por ejemplo, Heinrich, 2006, y Basilea II, 2001).

El desarrollo de técnicas para la medición y administración de riesgo operacional es muy dinámico. Hay, sin embargo, algunas prácticas comunes surgidas entre algunos bancos. La naturaleza dinámica de las técnicas implicadas se pueden dar por dos razones: el hecho de que el acuerdo de Basilea permite un grado sustancial de flexibilidad (en el contexto de unos criterios estrictos de clasificación) en el método utilizado para evaluar los requisitos de capital, especialmente en el método de medición avanzada (AMA, por sus siglas en inglés). La medición y administración de riesgo operacional hace frente a muchos desafíos, entre los cuales están el relativo corto periodo de tiempo de datos históricos de pérdidas, el rol del ambiente de control interno - el cual naturalmente cambia y hace que la pérdida de los datos históricos sea irrelevante-, el importante papel de los eventos de poca frecuencia, pero con pérdidas muy grandes. Estos desafíos son manejados con varios enfoques en la práctica. El estudio de los métodos de riesgo operacional trata de separar modelos que se utilizan para cuantificar el riesgo operacional y el capital económico de aquellos que se utilizan solamente para la administración del riesgo. Esta separación, sin embargo, es muy difícil de efectuar, pues el modelo usado para cuantificar el riesgo operacional puede también ser usado por su administración. Lo que en la práctica se hace es separar los modelos que son usados por la administración de riesgos operacionales internamente de los que son usados para el cálculo del capital económico para propósitos regulatorios. Llamamos al primer grupo modelos de administración de riesgo operacional; al segundo grupo se le denomina modelos para cuantificación y asignación de capital. Hay básicamente tres diferentes modelos para la cuantificación y asignación de capital dentro del método de medición avanzada: enfoque de distribución de pérdidas, enfoque de control y enfoque de escenario-básico.

Las principales diferencias entre estos enfoques es el énfasis de los elementos comunes. El enfoque de distribución de pérdidas enfatiza el uso de pérdida de datos internos, el enfoque de control enfatiza la evaluación del entorno empresarial y el sistema de control interno, mientras que el enfoque de escenario-básico usa varios escenarios para evaluar el riesgo de la organización. A pesar de las diferencias en el énfasis, en la práctica la mayoría de los bancos está tratando de usar los elementos de estos tres enfoques. 
El enfoque de distribución de pérdidas (LDA, por sus siglas en inglés) comienza con la recopilación de datos sobre pérdidas que es el indicador más objetivo de riesgos actualmente disponible. Con estos datos se estima la distribución de pérdidas; sin embargo, éstos proporcionan solamente una retrospectiva y, por lo tanto, no refleja necesariamente los cambios en el riesgo actual, ambiente de control y la pérdida de datos; en segundo lugar, no siempre está disponible en cantidades suficientes en cualquier institución financiera para permitir una evaluación razonable de exposición.

El LDA se ocupa de estas deficiencias mediante la integración de otros elementos de la AMA como datos externos, análisis de escenarios y factores que reflejen el entorno empresarial y el sistema de control interno; usa técnicas actuariales para modelar el comportamiento de las pérdidas operacionales de una empresa a través de la estimación de la frecuencia y severidad para producir una estimación objetiva de las pérdidas esperadas e inesperadas. Se inicia con la recolección de los datos de pérdidas para modelar la frecuencia y la severidad de éstas por separado; después, se agregan estas distribuciones a través de simulación Monte Carlo o técnicas estadísticas para obtener una distribución de las pérdidas totales de cada tipo de pérdida. Algunas distribuciones estadísticas de uso común son la de Poisson, distribución binomial negativa, Weibull y Gumbel para la frecuencia; y Lognormal, Gamma y la distribución logarítmica normal gamma para la severidad. El último paso consiste en ajustar una curva a la distribución de pérdidas totales obtenidos; para comprobar la bondad de ajuste se emplean técnicas estadísticas estándar como el de Pearson, Chi-cuadrado y el test de Kolmogorov-Smirnov.

Con el nuevo acuerdo de Basilea II, los bancos están obligados a calcular el capital económico; esto es cierto para los tres enfoques en la AMA. La diferencia entre el valor de la pérdida correspondiente al percentil en la cola y la media de la distribución de la pérdida total es el capital económico de los percentiles elegidos. La media se llama la pérdida esperada y la diferencia pérdida inesperada, de la que los bancos están obligados a protegerse.

En resumen:

1) Los métodos top-down de indicador simple. Este método fue elegido por el comité de Basilea como una primera aproximación al cálculo del riesgo operacional. Un solo indicador como el ingreso total de la institución, o la 
volatilidad del ingreso o los gastos totales se pueden considerar como el cargo total a cubrir por este riesgo.

2) Los métodos bottom-up que incluyen el juicio de un experto. La base para el análisis de un experto es un conjunto de escenarios. Los expertos identifican los riesgos y sus probabilidades de ocurrencia.

3) Medición interna. El Comité de Basilea propone el método de medición interna como un procedimiento más avanzado para calcular el costo de capital regulatorio.

4) Enfoque estadístico clásico. Análogamente a lo que se ha utilizado en los métodos de cuantificación para el riesgo de mercado y, más recientemente, el riesgo de crédito, también se ha avanzado en la investigación relativa a los métodos de cálculo para el riesgo operacional. Sin embargo, contrario a lo que sucede con el riesgo de mercado, es muy difícil encontrar un método estadístico ampliamente aceptado.

5) Modelos causales. Como alternativa a la estadística clásica surgen los modelos causales, que suponen dependencia entre eventos de riesgo; en otras palabras, cada evento representa una variable aleatoria (discreta o continua) con función de distribución condicional. Los eventos que no cuenten con registros históricos o bien que la calidad de los mismos no sea la requerida, se recurre a la opinión o juicio de los expertos para determinar las probabilidades condicionales de ocurrencia. La herramienta para modelar esta causalidad son las RB, las cuales se fundamentan en el teorema de Bayes y la topología de redes; esta herramienta es la que se desarrolla y aplica en este trabajo.

\section{Marco teórico}

En esta sección se presenta la teoría que soporta el desarrollo del trabajo. Se inicia con una discusión sobre el VaR condicional como medida de riesgo coherente en el sentido de Artzner et al. (1998). Después se utiliza el enfoque bayesiano para la construcción de RB, destacando sus ventajas con respecto del enfoque clásico en el estudio del RO. 


\section{Valor en Riesgo Condicional (CVaR)}

De acuerdo con Panjer (2006), el CVaR (del término en inglés Conditional Value at Risk) o Expected Shortfall (ES, por sus siglas en inglés) es una medida alternativa al VaR que cuantifica las pérdidas que se pueden encontrar en las colas de las distribuciones. Se define como la pérdida esperada para los casos en donde la pérdida de valor del portafolio exceda el valor del VaR.

Si $X$ denota la variable aleatoria de pérdida, el CVaR de $X$ a un nivel de confianza del $(1-p) \times 100 \%$, denotado por $\operatorname{CVaRp}(X)$, es la pérdida esperada dado que las pérdidas totales exceden el cuantil 100p de la distribución de $X$. Para distribuciones arbitrarias se puede escribir $\operatorname{CVaRp}(X)$ como:

$$
\mathrm{CVaR}_{p}(X)=\mathrm{E}\left[X \mid X>x_{p}\right]=\frac{\int_{x_{p}}^{\infty} x \mathrm{~d} F(x)}{1-F\left(x_{p}\right)}
$$

donde $F(x)$ es la función de distribución acumulada de $X$. Además, para distribuciones continuas se puede usar la función de densidad para escribir lo anterior como:

$$
\mathrm{CVaR}_{p}(X)=\mathrm{E}\left[X \mid X>x_{p}\right]=\frac{\int_{x_{p}}^{\infty} x f(x) d x}{1-F\left(x_{p}\right)}=\frac{\int_{p}^{1} \operatorname{VaR}_{u}(x) \mathrm{d} u}{1-p} .
$$

Así, el CVaR se puede ver como el promedio de todos los valores VaR sobre el nivel de confianza $p$. Además CVaR puede escribirse como:

$$
\mathrm{CVaR}_{p}(X)=\mathrm{E}\left[X \mid X>x_{p}\right]=x_{p}+\frac{\int_{x_{p}}^{\infty}\left(x-x_{p}\right) d F(x)}{1-F\left(x_{p}\right)}=\operatorname{VaR}_{p}(x)+e\left(x_{p}\right)
$$

donde $e\left(x_{p}\right)$ es la media de excesos de la función de pérdidas. ${ }^{7}$

${ }^{7}$ Para un análisis completo sobre la no coherencia del VaR, se recomienda revisar Venegas-Martínez (2006). 


\section{El paradigma bayesiano}

En el análisis estadístico existen dos paradigmas: el frecuentista y el bayesiano. La diferencia fundamental se relaciona con la definición de probabilidad. Los frecuentistas señalan que la probabilidad de un evento es el límite de su frecuencia relativa en el largo plazo, mientras que los bayesianos sostienen que la probabilidad es subjetiva, un nivel de creencias que se actualiza con la incorporación de nueva información; la probabilidad subjetiva (creencias) fundamentadas en una base de conocimientos constituye la probabilidad a priori; la probabilidad a posteriori representa la actualización de las creencias.

Un tomador de decisiones bayesiano aprende y revisa sus creencias con base en la nueva información que tenga disponible. Desde este punto de vista, las probabilidades son interpretadas como niveles de creencias. Por lo tanto, el proceso de aprendizaje bayesiano consiste en estar revisando y actualizando probabilidades. El teorema de Bayes es el medio formal para poner en práctica lo anterior. ${ }^{8}$

\section{Teorema de Bayes}

El teorema de Bayes es una regla que puede ser utilizada para actualizar creencias con base en nueva información (por ejemplo, datos observados). Si se denota por $E$ como la evidencia y se supone que un experto cree que se puede asociar con una probabilidad $P(E)$, el teorema de Bayes (TB) dice que después de observar los datos, $D$, las creencias sobre $E$ son ajustadas de acuerdo con la siguiente expresión:

donde

$$
P(E \mid D)=\frac{P(D \mid E) P(E)}{P(D)},
$$

1) $P(D \mid E)$ es la probabilidad condicional de los datos, dado que la evidencia a priori, $D$, es cierta.

2) $P(D)$ es la probabilidad incondicional de los datos, $P(D)>0$; también se puede expresar como:

$$
P(D)=P(D \mid E) P(E)+P\left(D \mid E^{C}\right) P\left(E^{C}\right) .
$$

${ }^{8}$ Para una revisión del teorema de Bayes ver, por ejemplo, Zellner (1971). 
La probabilidad de $E$, antes de tener los datos $P(E)$, es llamada probabilidad a prio$r i$, una vez actualizada $P(E \mid D)$ es denominada probabilidad a posteriori.

Se reescribe la forma continua del TB de la siguiente manera:

$$
P(\theta \mid \mathbf{y}) \propto L(\theta \mid \mathbf{y}) \pi(\theta \mid)
$$

donde $\theta$ es un parámetro desconocido por estimar, $\mathbf{y}$ es un vector de observaciones, registradas, $\pi(\theta)$ es una distribución a priori que depende de uno o más parámetros, denominados hiper-parámetros, $L(\theta \mid \mathbf{y})$ es la función de verosimilitud para $\theta$ y $P(\theta \mid y)$ es la distribución a posteriori de $\theta$ (actualización de la a priori). Dos preguntas surgen de lo anterior: ¿cómo traducir la información $a$ priori en su forma analítica $\pi(\theta)$ ?, ¿qué tan sensible es la inferencia a posteriori, a la selección de la a priori? Cabe destacar que estas preguntas han sido un amplio tema de interés en la literatura bayesiana ${ }^{9}$.

\section{Inferencia bayesiana}

La distribución a posteriori —una combinación de datos y de la distribución a priori- del parámetro o vector de parámetros $\theta$, dada la información $\mathbf{y}$, denotada por $P(\theta \mid \mathbf{y})$, es obtenida a través de la aplicación del teorema de Bayes y contiene la información relevante sobre el parámetro desconocido.

\section{Redes bayesianas}

Una red bayesiana (RB) es una gráfica que representa el dominio de las variables de decisión, las relaciones cuantitativas y cualitativas de éstas y sus medidas de probabilidad; asimismo, puede incluir funciones de utilidad que representan las preferencias del tomador de decisiones.

Una importante característica de las RB es su forma gráfica, lo cual permite representar en una forma visual complicados razonamientos probabilísticos. Otro aspecto por destacar es su parte cuantitativa porque permiten incorporar elementos subjetivos como lo son la opinión de expertos, así como probabilidades basadas en

${ }^{9}$ Ver Ferguson (1973). 
datos estadísticos. Tal vez la característica más importante es que son una representación directa del mundo real y no un proceso de razonamiento.

Las redes bayesianas son gráficas dirigidas acíclicas (GDA). Una gráfica es definida como un conjunto de nodos unidos por arcos. Si entre cada par de nodos hay una relación de precedencia representada por arcos, entonces la gráfica es dirigida. Un ciclo es una trayectoria que inicia y termina en el mismo nodo. Una trayectoria es una serie de nodos contiguos conectados por arcos dirigidos. Cada nodo en una RB se asocia con un conjunto de tablas de probabilidades. Los nodos representan las variables de interés, las cuales pueden ser discretas o continuas. Una red causal, de acuerdo con Pearl (2000), es una RB con la propiedad adicional de que los nodos padres son las causas dirigidas. ${ }^{10}$

\section{Algoritmos para cálculo de inferencia en redes bayesianas}

Una red bayesiana es empleada básicamente para inferencia a través del cálculo de las probabilidades condicionales dada la información disponible hasta el momento para cada nodo (creencias). Existen dos clases de algoritmos para el proceso de inferencia: el primero genera una solución exacta y el segundo produce una solución aproximada con alta probabilidad. Entre los algoritmos de inferencia exacta se tienen, por ejemplo, polytree, clique tree, junction tree, algorithms of variable elimination y method of Pear.

El uso de soluciones aproximadas es motivado por el crecimiento exponencial de tiempo de procesamiento requerido para soluciones exactas, de acuerdo con Guo y Hsu (2002) este tipo de algoritmos puede agruparse en stochastic simulation, model simplification methods, search based methods y loopy propagation methods; el más conocido es el de simulación estocástica que se divide en importance sampling algorithms y Markov Chain Monte Carlo (MCMC) methods.

\section{Construcción de una red bayesiana para el proceso de bancarización de Pro- campo}

En la gráfica 1 se muestra un flujograma simple para construir una RB. El primer paso es definir el dominio del problema donde se especifique el propósito de la

${ }^{10}$ Para una revisión de la teoría de las redes bayesianas consulte Jensen (1996). 
RB. Posteriormente se identifican las variables o nodos importantes en el dominio del problema. Después se representa en forma gráfica la interrelación entre nodos o variables. El modelo resultante debe ser validado por los expertos en el tema. En caso de haber desacuerdo entre ellos se regresa a uno de los pasos anteriores hasta alcanzar el consenso. Los últimos tres pasos son incorporar la opinión de los expertos (referida como la cuantificación de la red), crear escenarios factibles con la red (aplicaciones de redes) y poner a punto las estimaciones en el tiempo (mantenimiento de la red).

\section{Gráfica 2 \\ Diagrama de flujo RB}

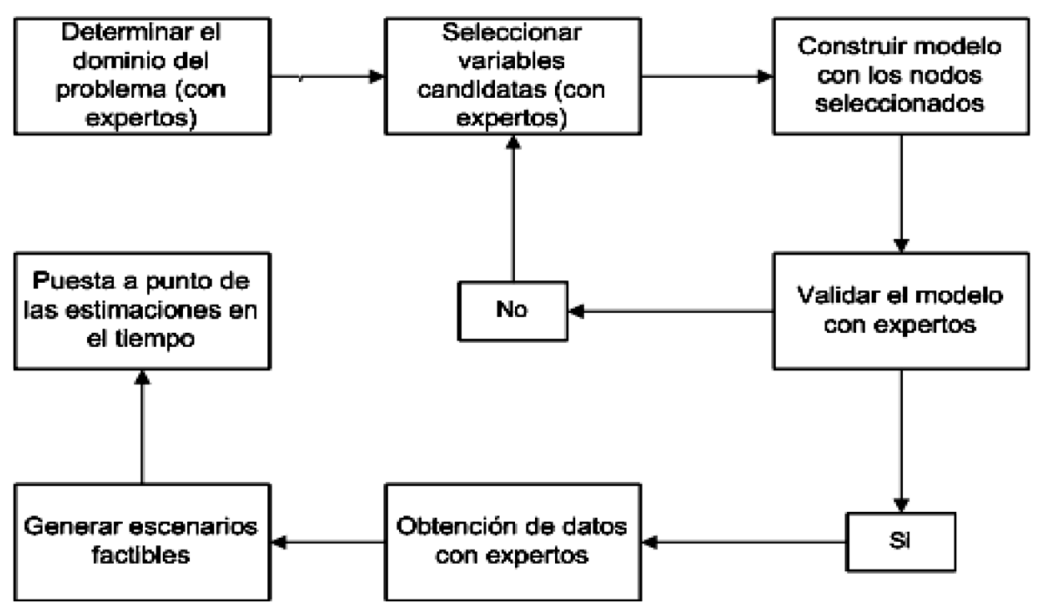

\section{Problemática}

Los principales problemas a que se enfrenta un administrador de riesgo que emplea RB son ¿cómo implementar una red bayesiana?, ¿cómo modelar su estructura?, ¿cómo cuantificarla?, ¿cómo utilizar datos subjetivos (de expertos) u objetivos (estadísticos) o ambos?, ¿qué instrumentos se deben utilizar para obtener mejores resultados?, ¿cómo validar el modelo? Las respuestas a estos cuestionamientos, en su momento, se abordarán en la aplicación del modelo bayesiano.

El objetivo principal de la aplicación consiste en elaborar una guía para implementar una RB para medir el riesgo operacional en el proceso de bancarización de Procampo. Asimismo, se pretende generar una medida consistente del capital 
económico necesario para hacer frente a pérdidas derivadas de eventos de riesgo operacional.

\section{Alcances de la aplicación}

El caso de estudio se enfoca en el análisis de los eventos de riesgo operacional que se presentaron durante la dispersión de recursos para el pago de apoyos de Procampo por parte de los bancos contratados para este servicio. Una vez identificados los factores de riesgo asociados a cada fase del proceso, se definen los nodos que formaran parte de la red bayesiana y que son variables aleatorias que pueden ser discretas o continuas, las cuales tienen asociadas distribuciones de probabilidad.

Para el caso de que se tengan datos históricos relacionados con los nodos (variables aleatorias) se les ajusta una función de distribución; en caso contrario, se recurre a los expertos o fuentes externas para determinar las probabilidades de ocurrencia o el parámetro de alguna función de probabilidad conocida. Los datos disponibles son quincenales y abarcan el periodo de 2008 a 2011; se calcula la máxima pérdida esperada quincenal derivada de fallas en el proceso de pagos de Procampo por trasferencia electrónica.

\section{Construcción y cuantificación del modelo}

Los nodos seleccionados son conectados con arcos dirigidos (con flechas) para formar una estructura que muestra la dependencia o relación causal entre éstos. La RB se divide en dos redes: una para modelar la frecuencia y la otra para la severidad. Lo anterior facilita su análisis, pues una vez obtenidos los resultados por separado se agregan a través del método de simulación Monte Carlo para obtener la pérdida esperada.

\section{Frecuencia}

La red completa de la frecuencia se muestra en la gráfica 3, que se genera a partir del análisis de factores de riesgo vinculados con la bancarización. Estos factores se presentan durante el proceso de dispersión de recursos (transferencias electrónicas a las cuentas de beneficiarios) que se opera a través del sector bancario. Los riesgos se clasificaron de la siguiente manera: 
a) Sistemas

I. Cancelación del servicio

II. Causa indefinida

III. Cuenta bloqueada

b) Recursos humanos

I. Insuficiencia de fondos

II. Cuenta inexistente

III. Cuenta no pertenece al banco receptor

c) Procesos

I. Cuenta cancelada

II. Cuenta en otra divisa

III. Cuenta inexistente

Gráfica 3

Red para frecuencia de fallas

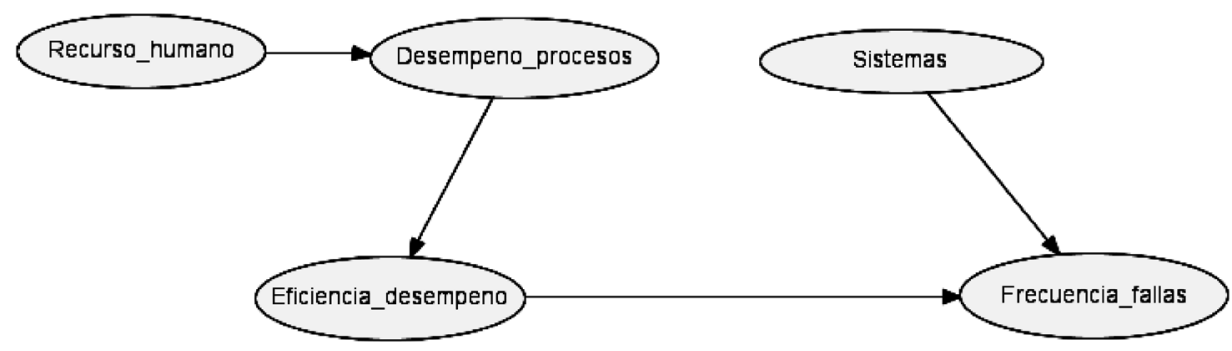

Severidad

La red de la severidad se muestra en la gráfica 4, que se integra por cuatro nodos, aunque requiere una cantidad importante de probabilidades. El nodo etiquetado como severidad de pérdida es el costo adicional que se incurre por no realizar las trasferencias de manera exitosa, lo que obliga a pagar a través de cheques; los otros nodos se consideran variables informativas. 


\section{Gráfica 4}

Red para severidad de fallas

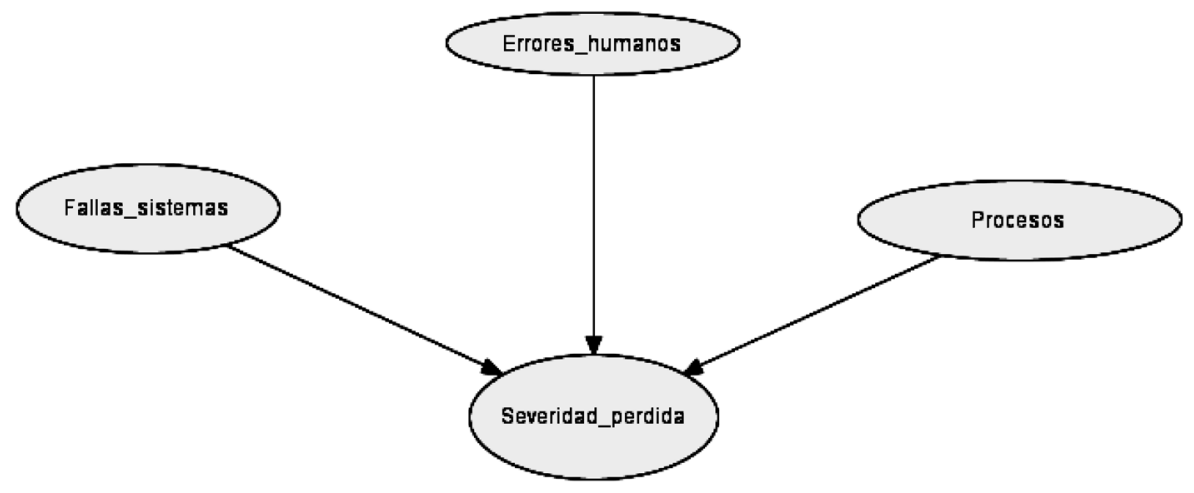

A continuación se describen las características y estados de cada nodo de las redes de severidad y frecuencia respectivamente.

\section{Cuadro 2}

\section{Nodos de la red de severidad}

\begin{tabular}{|c|c|c|}
\hline Nodo & Descripción & Estados \\
\hline \multirow{4}{*}{ Falla en sistemas } & \multirow{4}{*}{$\begin{array}{l}\text { Fallas en los sistemas } \\
\text { de información }\end{array}$} & 0 a 500 \\
\hline & & 500 a 1000 \\
\hline & & 1000 a 1500 \\
\hline & & $>1500$ \\
\hline \multirow{5}{*}{$\begin{array}{c}\text { Errores } \\
\text { humanos }\end{array}$} & \multirow{5}{*}{$\begin{array}{c}\text { Errores humanos que } \\
\text { se traducen en pérdidas }\end{array}$} & 0 a 500 \\
\hline & & 500 a 1000 \\
\hline & & 1000 a 2000 \\
\hline & & 2000 a 3500 \\
\hline & & $>3500$ \\
\hline \multirow{4}{*}{$\begin{array}{l}\text { Fallas en los } \\
\text { procesos }\end{array}$} & \multirow{4}{*}{$\begin{array}{c}\text { Deficiencias en } \\
\text { procesos que evitan el } \\
\text { pago de apoyos }\end{array}$} & 0 a 5000 \\
\hline & & 5 a 15000 \\
\hline & & 15000 a 35000 \\
\hline & & $>35000$ \\
\hline \multirow{5}{*}{$\begin{array}{l}\text { Severidad de } \\
\text { pérdidas }\end{array}$} & \multirow{5}{*}{$\begin{array}{c}\text { Pérdida esperada por } \\
\text { eventos de riesgo } \\
\text { operacional }\end{array}$} & 0 a 5000 \\
\hline & & 5000 a 10000 \\
\hline & & 10000 a 30000 \\
\hline & & 30000 a 50000 \\
\hline & & $>50000$ \\
\hline
\end{tabular}




\section{Cuadro 3}

\section{Nodos de la red de frecuencia}

\begin{tabular}{|c|c|c|}
\hline Nombre nodo & Descripción del nodo & Estados \\
\hline \multirow{3}{*}{$\begin{array}{l}\text { Recurso } \\
\text { humano }\end{array}$} & \multirow{3}{*}{$\begin{array}{l}\text { Errores humanos que se } \\
\text { traducen en no pagos }\end{array}$} & Insuficiencia de recursos en cuenta \\
\hline & & Cuenta no pertenece al banco receptor \\
\hline & & Orden de no pagar al emisor \\
\hline \multirow{3}{*}{$\begin{array}{l}\text { Desempeño de } \\
\text { procesos }\end{array}$} & \multirow{3}{*}{$\begin{array}{l}\text { Fallas de procesos de } \\
\text { pago }\end{array}$} & Cancelación de cuenta \\
\hline & & Cuenta en otra divisa \\
\hline & & Cuenta inexistente \\
\hline \multirow{3}{*}{$\begin{array}{l}\text { Eficiencia en } \\
\text { desempeño }\end{array}$} & \multirow{3}{*}{$\begin{array}{l}\text { Eficiencia del desempeño } \\
\text { de personas y procesos }\end{array}$} & Excelente \\
\hline & & Promedio \\
\hline & & Mal \\
\hline \multirow{3}{*}{$\begin{array}{l}\text { Fallas en } \\
\text { sistemas }\end{array}$} & \multirow{3}{*}{$\begin{array}{l}\text { Fallas en los sistemas } \\
\text { que procesan las órdenes } \\
\text { de pago }\end{array}$} & Cancelación del servicio \\
\hline & & Causa indefinida \\
\hline & & Bloqueo de cuentas \\
\hline \multirow{5}{*}{$\begin{array}{l}\text { Frecuencia de } \\
\text { fallas }\end{array}$} & \multirow{5}{*}{$\begin{array}{l}\text { Número de fallas en } \\
\text { un periodo de tiempo. } \\
\text { Retrasos, pagos } \\
\text { incorrectos, pagos mal } \\
\text { direccionados, sin pago, } \\
\text { pagos duplicados }\end{array}$} & 0 a 500 \\
\hline & & 500 a 1000 \\
\hline & & 1000 a 2000 \\
\hline & & 2000 a 3000 \\
\hline & & Más de 3000 \\
\hline
\end{tabular}

\section{Cuantificación de la red bayesiana}

Para cuantificar las redes bayesianas identificadas en la sección anterior, se utilizaron tanto datos objetivos como subjetivos. A continuación se describen las herramientas o técnicas que se emplearon para obtener, codificar y cuantificar los tipos de datos.

\section{- Análisis estadístico de la red de frecuencia}

En este apartado se analiza cada nodo de la red de frecuencia; para el caso de nodos con información histórica disponible, se ajusta ${ }^{11}$ la distribución de probabilidades correspondiente y se calculan las probabilidades requeridas. Cuando no se

\footnotetext{
${ }^{11}$ Ajustar una distribución consiste en encontrar una función matemática que represente de la mejor manera a una variable estadística. Existen cuatro pasos para llevar a cabo el ajuste: 1) hipótesis sobre el modelo, 2) estimación de parámetros, 3) evaluación de la calidad de ajuste y 4) prueba estadística de la bondad de ajuste. Para realizar este trabajo se utilizó el lenguaje estadístico R: primero se graficó la distribución de frecuencias de los datos reales para proponer un modelo de distribución, después se realizaron diferentes estimaciones para encontrar el mejor parámetro; finalmente se realizó la prueba Kolmogorov-Smirnov (K-S) para determinar estadísticamente la bondad del ajuste, que será un buen indicador si p-value $>0.05$.
} 
cuenta con datos suficientes se obtiene la información de los expertos o de fuentes externas. Los errores ocasionados por el personal interno en el desempeño de sus funciones denotado por el nodo Recurso humano tiene la siguiente distribución de probabilidades:

\section{Cuadro 4 \\ Probabilidades Recurso humano}

\begin{tabular}{l|l}
\hline \multicolumn{2}{c}{ Probabilidades } \\
\hline Insuficiencia de recursos & 0.85 \\
\hline Cuenta no pertenece al emisor & 0.1 \\
\hline Orden de no pagar al emisor & 0.05 \\
\hline
\end{tabular}

Fuente: Elaboración propia con información de expertos o externa.

El buen funcionamiento de las instituciones bancarias depende del desempeño de sus procesos; la madurez de éstos se asocia a sistemas de gestión de la calidad en el nivel proceso y producto. Las probabilidades condicionales del nodo etiquetado como Desempeño de procesos son las siguientes:

\section{Cuadro 5}

\section{Probabilidades Desempeño de procesos}

\begin{tabular}{c|c|c|c}
\hline \multicolumn{4}{c}{ Desempeño de procesos } \\
\hline $\begin{array}{c}\text { Recurso } \\
\text { humano }\end{array}$ & $\begin{array}{c}\text { Insuficiencia } \\
\text { de recursos }\end{array}$ & $\begin{array}{c}\text { Cuenta no pertenece al } \\
\text { emisor }\end{array}$ & $\begin{array}{c}\text { Orden de no } \\
\text { pagar al emisor }\end{array}$ \\
\hline $\begin{array}{c}\text { Cuenta } \\
\text { cancelada }\end{array}$ & 0.82 & 0.8 & 0.7 \\
\hline $\begin{array}{c}\text { Cuenta en otra } \\
\text { divisa }\end{array}$ & 0.09 & 0.1 & 0.2 \\
\hline $\begin{array}{c}\text { Cuenta } \\
\text { inexistente }\end{array}$ & 0.09 & 0.1 & 0.1 \\
\hline
\end{tabular}

Fuente: Elaboración propia con información de expertos o externa.

Con respecto a las probabilidades del nodo etiquetado como Eficiencia del desempeño son condicionadas a las probabilidades de los nodos Desempeño de procesos y Recurso humano, con lo cual se obtiene la siguiente tabla de probabilidades condicionales: 


\section{Cuadro 6}

\section{Probabilidades condicionales Eficiencia del desempeño}

\begin{tabular}{c|c|c|c|c|c|c|c|c|c}
\hline \multicolumn{10}{c|}{ Eficiencia del desempeño } \\
\hline Recurso H. & \multicolumn{2}{|c|}{ Insuficiencia recursos } & \multicolumn{3}{c|}{$\begin{array}{c}\text { Cta no pertenece } \\
\text { emisor }\end{array}$} & \multicolumn{3}{c}{ Orden no pagar emisor } \\
\hline $\begin{array}{c}\text { Desempeño } \\
\text { procesos }\end{array}$ & CC & COD & CI & CC & COD & CI & CC & COD & CI \\
\hline Excelente & 0.2 & 0.16 & 0.65 & 0.2 & 0.16 & 0.65 & 0.2 & 0.16 & 0.65 \\
\hline Promedio & 0.1 & 0.16 & 0.21 & 0.1 & 0.16 & 0.21 & 0.1 & 0.16 & 0.21 \\
\hline Mal & 0.7 & 0.66 & 0.13 & 0.7 & 0.66 & 0.13 & 0.7 & 0.66 & 0.13 \\
\hline
\end{tabular}

Fuente: Elaboración propia con información de expertos y externa.

Existe una probabilidad condicional del $20 \%$ — dado que por error del recurso humano no se tengan recursos presupuestales suficientes y que por proceso se cancelé la cuenta - de que el desempeño sea excelente. Las otras probabilidades condicionales se leen de manera similar.

Con respecto al nodo etiquetado como Sistemas, son errores generados por los sistemas de información del banco que tienen la siguiente distribución de probabilidades que se calcularon con información cualitativa proporcionada por los expertos en los sistemas de pago de Aserca.

\section{Cuadro 7}

\section{Probabilidades Sistemas}

\begin{tabular}{c|c}
\hline \multicolumn{2}{c}{ Probabilidades } \\
\hline Cancelación del servicio & 0.52 \\
\hline Causa indefinida & 0.41 \\
\hline Cuenta bloqueada & 0.07 \\
\hline
\end{tabular}

Fuente: Elaboración propia con información de expertos o externa.

Finalmente, para el nodo objetivo Frecuencia de fallas, se supone una función de distribución binomial negativa con probabilidad de éxito $p=0.51$ y límite de éxitos igual a 1.000; este supuesto es consistente con la práctica financiera y estudios del riesgo operacional que muestran que el número de fallas usualmente siguen una distribución de Poisson o una binomial negativa. Para estimar el valor de los parámetros se recurrió a los expertos y se complementó con resultados del análisis de los otros nodos de la red de frecuencia. 
- Análisis estadístico de la red de severidad

En este apartado se analiza cada nodo de la red de severidad. Para el caso de nodos con información histórica disponible se ajusta la distribución de probabilidad correspondiente y se calculan las posibilidades requeridas. Cuando no se cuenta con datos suficientes se obtiene la información de los expertos o externa.

El nodo Falla en sistemas presenta la siguiente distribución de frecuencias y densidad exponencial ajustada para las pérdidas ocasionadas por fallas en los sistemas.

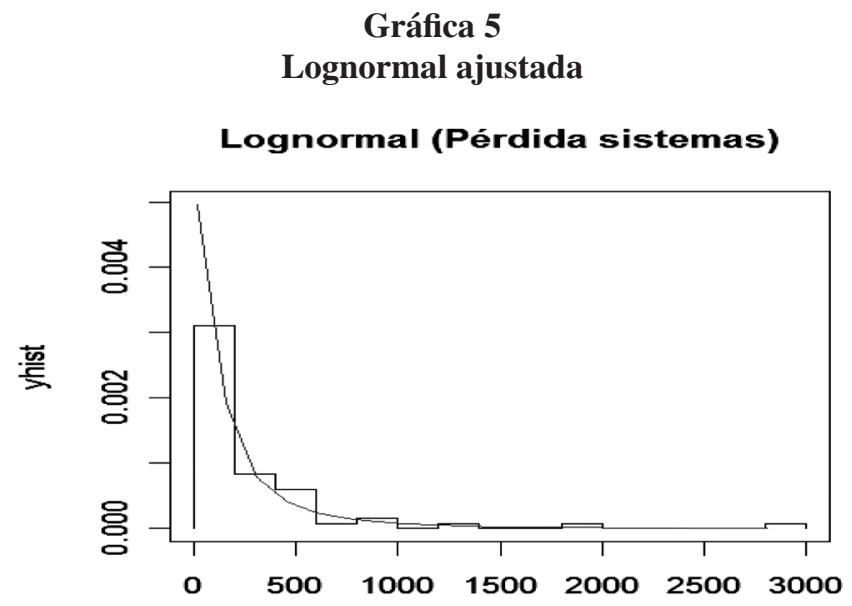

Para determinar la bondad del ajuste se realizó la prueba de Kolmogorov-Smirnov con los siguientes valores: $D=0.0811, p$-value $=0.77$. En otras palabras se acepta la hipótesis nula de que la muestra proviene de una Lognormal. Por lo tanto, se calcula la tabla de probabilidad para este nodo de la red de severidad misma que constituye las probabilidades a priori.

\section{Cuadro 8}

Probabilidades Falla en sistema

\begin{tabular}{l|l}
\hline \multicolumn{2}{c}{ Falla en sistema } \\
\hline 0 a 500 & 0.86 \\
\hline 500 a 1000 & 0.09 \\
\hline 1000 a 1500 & 0.025 \\
\hline$>1500$ & 0.025 \\
\hline
\end{tabular}


A partir del cuadro se ve que existe una probabilidad del $86 \%$ de que se pierdan hasta 500 pesos por fallas en los sistemas, $9 \%$ de probabilidad de que se pierdan entre 500 y 1000 pesos, $2.5 \%$ de la pérdida está entre 1000 y 1500 pesos, y $2.5 \%$ de probabilidad de que la pérdida sea mayor a 1500 pesos en un periodo de 15 días.

A continuación se analiza el nodo Errores humanos, el cual presenta la siguiente distribución de frecuencia y densidad Lognormal ajustada para pérdidas ocasionadas por errores humanos.

\section{Gráfica 6 \\ Lognormal ajustada}

Lognormal (Pérdida errores humanos)

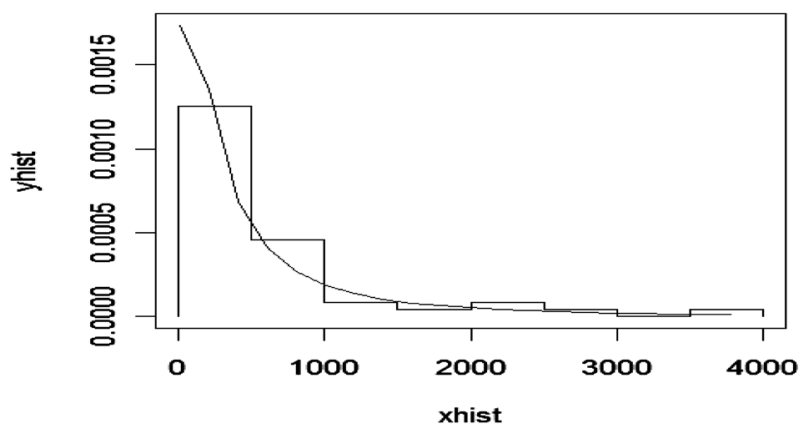

Para determinar la bondad del ajuste se realizó la prueba K-S con los siguientes resultados: $D=0.1064$ y $p$-value $=0.6486$. De esta manera se acepta la hipótesis nula de que la muestra proviene de una Lognormal. Por lo tanto, se calculan las probabilidades para este nodo de la red de severidad, las cuales constituyen las probabilidades a priori.

\section{Cuadro 9}

Probabilidades Errores humanos

\begin{tabular}{c|c}
\hline \multicolumn{2}{c}{ Errores humanos } \\
\hline 0 a 500 & 0.66 \\
\hline 500 a 1000 & 0.16 \\
\hline 1000 a 2000 & 0.10 \\
\hline 2000 a 3500 & 0.04 \\
\hline$>3500$ & 0.03 \\
\hline
\end{tabular}


A partir del cuadro se observa que existe una probabilidad del $66 \%$ de que se pierdan menos de 500 pesos por fallas en errores humanos, $16 \%$ de probabilidad de que se pierdan entre 500 y 1000 pesos, $10 \%$ de que la pérdida esté entre 1000 y 2000 pesos, $4 \%$ de que la pérdida esté entre 2000 y 3500 pesos, y $3 \%$ de probabilidad de que la pérdida sea mayor a 3500 pesos quincenales.

Para el nodo Procesos se presenta la siguiente distribución de frecuencias y densidad Lognormal ajustada para las pérdidas ocasionadas por fallas en los procesos o procedimientos, entre otros.

Gráfica 7

Lognormal ajustada

\section{Lognormal (Pérdida procesos)}

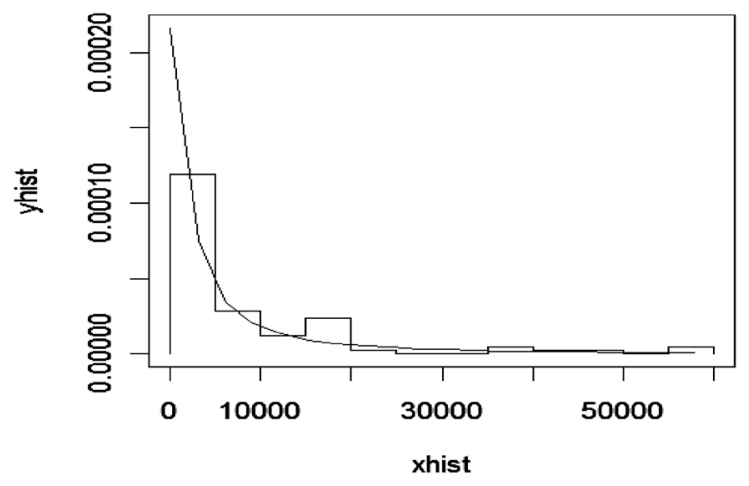

Para determinar la bondad del ajuste se realizó la prueba K-S que produce los siguientes valores: $\quad D=0.0799, p$-value $=0.6575$. En otras palabras se acepta la hipótesis nula de que la muestra proviene de una Lognormal; por lo tanto, se calcula la probabilidad para este nodo de la red de severidad que constituye las probabilidades a priori.

\section{Cuadro 10}

Procesos

\begin{tabular}{c|c}
\hline Pérdidas por procesos & Probabilidad \\
\hline 0 a 5000 & 0.63 \\
\hline 5000 a 15000 & 0.20 \\
\hline 15000 a 35000 & 0.10 \\
\hline > 35000 & 0.07 \\
\hline
\end{tabular}

Fuente: Elaboración propia con información de los expertos. 
De acuerdo con los datos generados existe una probabilidad del $63 \%$ de que se pierdan menos de 5000 pesos por eventos derivados en fallas en procesos, $20 \%$ de probabilidad de que se pierdan entre 5000 y 15000 pesos, $10 \%$ de que se pierdan entre 15000 y 35000 pesos, y 7\% de probabilidad de que la pérdida sea mayor a 35000 pesos en una quincena.

Por último, el nodo objetivo Severidad de la pérdida representa las pérdidas asociadas con los nodos Fallas en sistemas, Errores humanos y Eventos en procesos. Para el cálculo de la tabla de probabilidades condicional se utilizó una función de distribución Lognormal con parámetros media de $\log (x)=8.13$ y desviación estándar de 1.57. A continuación se generan las probabilidades a posteriori para lo cual se utilizan técnicas de inferencia bayesiana.

\section{Probabilidades a posteriori}

Una vez analizados cada uno de los nodos (variables aleatorias continuas o discretas) de las redes tanto de frecuencia como de severidad y asignadas las correspondientes funciones de distribución de probabilidades, se generan las probabilidades a posteriori; para ello se utilizan técnicas de inferencia para redes bayesianas, en particular el algoritmo denominado junction tree. ${ }^{12}$ Las probabilidades a posteriori para los nodos de la red de frecuencia que tienen al menos un padre ${ }^{13}$ son las siguientes.

\section{Gráfica 8}

Probabilidades a posteriori para la red de frecuencia

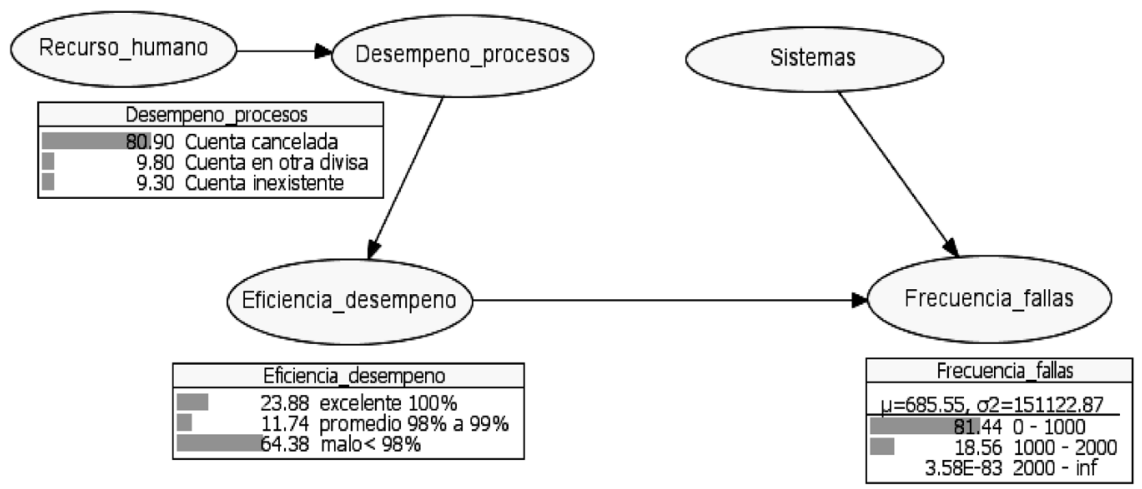

${ }^{12}$ Para una consulta detallada del algoritmo revise Guo y Hsu, 2002.

${ }^{13}$ Los nodos que no tienen padre mantienen las probabilidades a priori. 
Los resultados del nodo Eficiencia del desempeño muestran que existe una probabilidad del $24 \%$ de tener un desempeño excelente. Asimismo, una probabilidad del $12 \%$ de que se presenten problemas menores con un desempeño medio y una probabilidad del $64 \%$ de que el desempeño sea deficiente. Las probabilidades calculadas están condicionadas por el desempeño de los procesos.

En cuanto al nodo Desempeño de procesos, la probabilidad de que existan cuentas canceladas es del $81 \%$, de que la cuenta esté en otra divisa es del $10 \%$ y de que la cuenta no exista es del $9 \%$; todo esto condicionado a que existan fallas en el recurso humano.

Por último, la distribución de probabilidades del nodo de interés Frecuencia de fallas muestra una probabilidad del $81 \%$ de que se presenten menos de 1000 fallas, una probabilidad del $19 \%$ de que tengan entre 1000 y 2000 fallas, y una probabilidad nula de que se presenten más de 2000 fallas; dichas probabilidades están condicionadas a los equívocos de sistemas y de la eficiencia de los procesos y confiabilidad de las personas.

Para el cálculo de las probabilidades del nodo de interés se utilizó una binomial negativa con parámetros número de éxitos $=1000$ y probabilidad de éxito $p=0.51$, lo cual es consistente con la evidencia empírica de que la frecuencia de eventos de riesgo operacional tienen un ajuste adecuado bajo esta distribución. ${ }^{14}$ Para el caso de la red de severidad se tiene las siguientes probabilidades a posteriori.

${ }^{14}$ Ver Svetlozar et al. (2008). 


\section{Gráfica 9}

\section{Probabilidades a posteriori para la red de severidad}

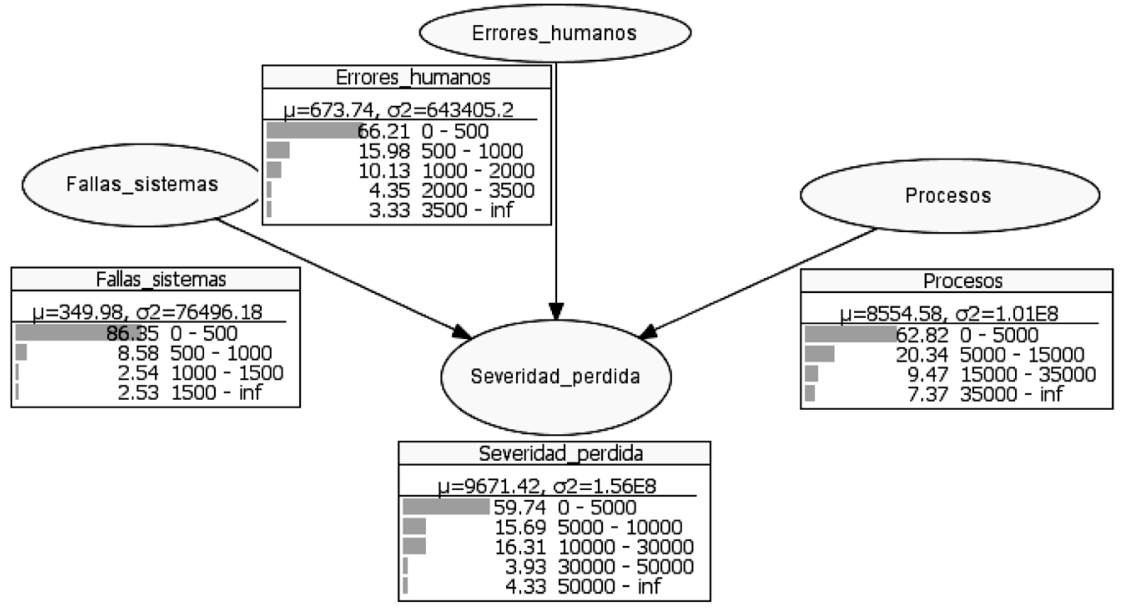

Las pérdidas originadas por errores humanos en promedio son de 673 pesos quincenales; por lo que respecta a las pérdidas por eventos en los procesos son de 8554 pesos quincenales en promedio. En cuanto a fallas en los sistemas en promedio se tiene una pérdida quincenal de 350 pesos. La distribución de probabilidades del nodo de interés Severidad de pérdida muestra una probabilidad del $59.7 \%$ de que la pérdida esté entre 0 y 5000 pesos, una probabilidad del $15.69 \%$ de que esté entre 5000 y 10000 pesos, una probabilidad del $16.31 \%$ de que esté entre 10000 y 30000 pesos, una probabilidad del 3.93\% de que esté entre 30000 y 50000 pesos, y una probabilidad del $4.33 \%$ de que la pérdida sea mayor a 50000 pesos quincenales.

\section{Cálculo del Valor en Riesgo Operacional (OpVaR)}

Una vez realizada la inferencia bayesiana para obtener las distribuciones de probabilidad a posteriori de la frecuencia y severidad de pérdidas, se integran ambas distribuciones para generar la distribución de pérdidas potenciales (se utilizó una binomial negativa para la frecuencia y una Lognormal para la severidad) a través de un proceso de simulación Monte Carlo. ${ }^{15}$

\footnotetext{
${ }^{15}$ Los resultados de la simulación están disponibles a los lectores mediante solicitud por correo electrónico.
} 
Para el cálculo del OpVar se ordenan los valores obtenidos para las pérdidas esperadas en orden descendente y se calculan los percentiles correspondientes:

\section{Cuadro 11}

\section{Percentiles para modelo bayesiano}

\begin{tabular}{|c|c|c|}
\hline Posición & Pérdida(pesos) & Porcentaje \\
\hline 5 & 14747.69 & $100.00 \%$ \\
\hline 21 & 14733.59 & $97.90 \%$ \\
\hline 25 & 14691.29 & $95.80 \%$ \\
\hline 24 & 14493.90 & $89.50 \%$ \\
\hline 40 & 14493.90 & $89.50 \%$ \\
\hline 45 & 14493.90 & $89.50 \%$ \\
\hline 35 & 14465.70 & $87.50 \%$ \\
\hline 4 & 14451.60 & $85.40 \%$ \\
\hline 44 & 14282.41 & $83.30 \%$ \\
\hline 17 & 14197.82 & $81.20 \%$ \\
\hline 37 & 14155.52 & $79.10 \%$ \\
\hline 10 & 14028.63 & $77.00 \%$ \\
\hline 18 & 13972.23 & $72.90 \%$ \\
\hline 22 & 13972.23 & $72.90 \%$ \\
\hline 12 & 13958.13 & $70.80 \%$ \\
\hline 9 & 13873.54 & $68.70 \%$ \\
\hline 6 & 13788.94 & $66.60 \%$ \\
\hline 27 & 13760.75 & $64.50 \%$ \\
\hline 49 & 13746.65 & $62.50 \%$ \\
\hline 47 & 13732.55 & $60.40 \%$ \\
\hline 38 & 13718.45 & $58.30 \%$ \\
\hline 2 & 13633.85 & $52.00 \%$ \\
\hline 14 & 13633.85 & $52.00 \%$ \\
\hline 30 & 13633.85 & $52.00 \%$ \\
\hline 19 & 13577.46 & $50.00 \%$ \\
\hline
\end{tabular}

\begin{tabular}{|c|c|c|}
\hline Posición & Pérdida(pesos) & Porcentaje \\
\hline 26 & 13549.26 & $47.90 \%$ \\
\hline 36 & 13535.16 & $45.80 \%$ \\
\hline 3 & 13506.96 & $43.70 \%$ \\
\hline 31 & 13492.86 & $41.60 \%$ \\
\hline 34 & 13464.66 & $39.50 \%$ \\
\hline 15 & 13394.17 & $35.40 \%$ \\
\hline 39 & 13394.17 & $35.40 \%$ \\
\hline 41 & 13365.97 & $33.30 \%$ \\
\hline 13 & 13337.77 & $31.20 \%$ \\
\hline 46 & 13210.88 & $29.10 \%$ \\
\hline 11 & 13196.78 & $27.00 \%$ \\
\hline 1 & 13041.69 & $25.00 \%$ \\
\hline 7 & 12999.39 & $20.80 \%$ \\
\hline 16 & 12999.39 & $20.80 \%$ \\
\hline 48 & 12971.20 & $18.70 \%$ \\
\hline 20 & 12943.00 & $14.50 \%$ \\
\hline 33 & 12943.00 & $14.50 \%$ \\
\hline 43 & 12886.60 & $12.50 \%$ \\
\hline 23 & 12858.40 & $10.40 \%$ \\
\hline 29 & 12816.10 & $8.30 \%$ \\
\hline 32 & 12703.31 & $6.20 \%$ \\
\hline 8 & 12238.04 & $4.10 \%$ \\
\hline 42 & 12012.45 & $2.00 \%$ \\
\hline 28 & 11941.96 & $0.00 \%$ \\
\hline
\end{tabular}

En consecuencia, si se considera calcular el OpVaR con un nivel de confianza del 96\%, se tiene una máxima pérdida esperada de 14691.29 pesos quincenales por riesgo operacional, relacionados con el proceso de bancarización de Procampo.

\section{Validación del modelo bayesiano}

Para validar los resultados del modelo bayesiano, se estiman con modelos clásicos la distribución de probabilidades para la frecuencia y la severidad. Posteriormente, a través de simulación Monte Carlo, se integran ambas distribuciones para obtener 
la distribución de pérdidas esperadas. Por último, se calcula el riesgo operacional con la distribución de pérdidas estimada en forma clásica y se comparan los resultados con los obtenidos con el modelo bayesiano.

\section{Análisis clásico de frecuencia}

Se considera el número de fallas que quincenalmente se presentan en la dispersión de los apoyos que se realizan a través de los principales bancos en México y se le ajusta una distribución binomial negativa con parámetro $\mu=477$, según se muestra en la siguiente gráfica.

\section{Gráfica 12}

\section{Binomial negativa ajustada}

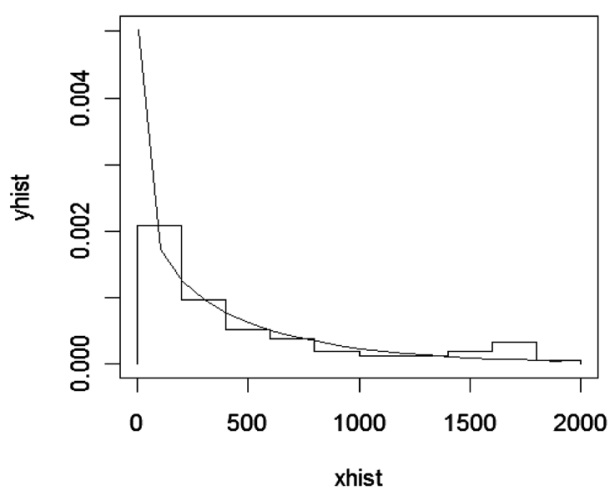

La prueba Kolmogorov-Smirnov tuvo los siguientes resultados: $\mathrm{D}=0.058$, $p$-value $=0.95$, lo que implica aceptar la hipótesis nula de que la muestra proviene de una distribución binomial negativa.

\section{Análisis clásico de severidad}

Como pérdidas se considera el monto quincenal que se paga por reexpedición del cheque debido a que no se realizó la trasferencia electrónica a la cuenta del beneficiario y se le ajusta una distribución Lognormal con parámetro media=7.58 y desviación estándar $=1.53$, según se muestra en la siguiente gráfica. 


\section{Gráfica 13}

Binomial negativa justada

\section{Lognormal (severidad bancarización)}

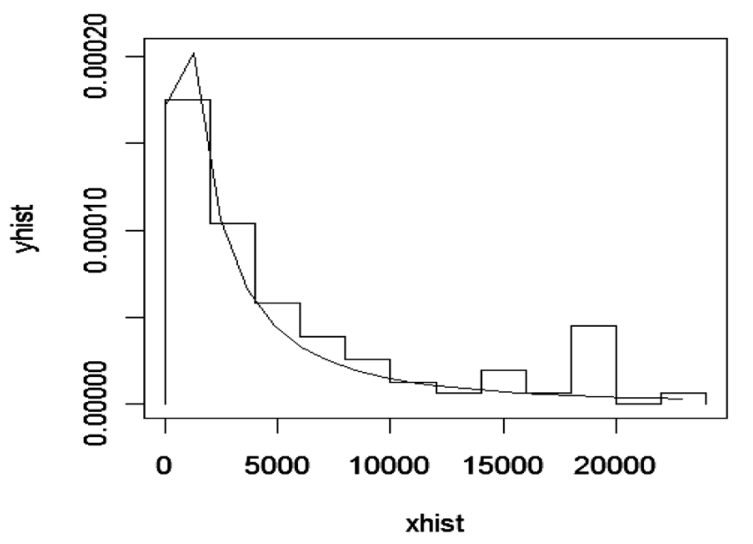

Se realizó la prueba K-S con los siguientes resultados: $\mathrm{D}=0.098$, $p$-value $=0.44$. El $p$-value es mayor al 5\%; por tanto, se acepta que la muestra proviene de una Lognormal.

\section{Cálculo del valor en riesgo operacional con el modelo clásico}

A través de Monte Carlo se integran las distribuciones clásicas de frecuencia y severidad para generar la distribución de pérdidas potenciales (se utilizó una binomial negativa para la frecuencia y una Lognormal). Para el cálculo del OpVar se ordenan los valores obtenidos para las pérdidas esperadas en orden descendente y se calculan los percentiles correspondientes; a continuación se muestran los resultados con niveles de confianza mayor a 96. 


\section{Cuadro 14}

\section{Percentiles para modelo clásico}

\begin{tabular}{|c|c|c|}
\hline Posición & Pérdida(pesos) & Porcentaje \\
\hline 5 & 5672.37 & $100.00 \%$ \\
\hline 21 & 5666.95 & $97.90 \%$ \\
\hline 25 & 5650.68 & $95.80 \%$ \\
\hline 24 & 5574.76 & $89.50 \%$ \\
\hline 40 & 5574.76 & $89.50 \%$ \\
\hline 45 & 5574.76 & $89.50 \%$ \\
\hline 35 & 5563.91 & $87.50 \%$ \\
\hline 4 & 5558.49 & $85.40 \%$ \\
\hline 44 & 5493.41 & $83.30 \%$ \\
\hline 17 & 5460.88 & $81.20 \%$ \\
\hline 37 & 5444.61 & $79.10 \%$ \\
\hline 10 & 5395.80 & $77.00 \%$ \\
\hline 18 & 5374.11 & $72.90 \%$ \\
\hline 22 & 5374.11 & $72.90 \%$ \\
\hline 12 & 5368.69 & $70.80 \%$ \\
\hline 9 & 5336.15 & $68.70 \%$ \\
\hline 6 & 5303.61 & $66.60 \%$ \\
\hline 27 & 5292.77 & $64.50 \%$ \\
\hline 49 & 5287.34 & $62.50 \%$ \\
\hline 47 & 5281.92 & $60.40 \%$ \\
\hline 38 & 5276.50 & $58.30 \%$ \\
\hline 2 & 5243.96 & $52.00 \%$ \\
\hline 14 & 5243.96 & $52.00 \%$ \\
\hline 30 & 5243.96 & $52.00 \%$ \\
\hline 19 & 5222.27 & $50.00 \%$ \\
\hline
\end{tabular}

\begin{tabular}{|c|c|c|}
\hline Posición & Pérdida(pesos) & Porcentaje \\
\hline 26 & 5211.42 & $47.90 \%$ \\
\hline 36 & 5206.00 & $45.80 \%$ \\
\hline 3 & 5195.15 & $43.70 \%$ \\
\hline 31 & 5189.73 & $41.60 \%$ \\
\hline 34 & 5178.89 & $39.50 \%$ \\
\hline 15 & 5151.77 & $35.40 \%$ \\
\hline 39 & 5151.77 & $35.40 \%$ \\
\hline 41 & 5140.93 & $33.30 \%$ \\
\hline 13 & 5130.08 & $31.20 \%$ \\
\hline 46 & 5081.27 & $29.10 \%$ \\
\hline 11 & 5075.85 & $27.00 \%$ \\
\hline 1 & 5016.20 & $25.00 \%$ \\
\hline 7 & 4999.93 & $20.80 \%$ \\
\hline 16 & 4999.93 & $20.80 \%$ \\
\hline 48 & 4989.08 & $18.70 \%$ \\
\hline 20 & 4978.24 & $14.50 \%$ \\
\hline 33 & 4978.24 & $14.50 \%$ \\
\hline 43 & 4956.55 & $12.50 \%$ \\
\hline 23 & 4945.70 & $10.40 \%$ \\
\hline 29 & 4929.43 & $8.30 \%$ \\
\hline 32 & 4886.05 & $6.20 \%$ \\
\hline 8 & 4707.09 & $4.10 \%$ \\
\hline 42 & 4620.33 & $2.00 \%$ \\
\hline 28 & 4593.21 & $0.00 \%$ \\
\hline
\end{tabular}

Si se calcula el OpVaR con un nivel de confianza del 96\%, se tiene una máxima pérdida esperada de 5650 pesos quincenales por riesgo operacional con el modelo clásico. Los resultados anteriores muestran que el OpVaR calculado con el modelo bayesiano es mayor al calculado con el modelo clásico, lo cual se explica por la causalidad entre los distintos factores de riesgo, la cual no está considerada en el modelo clásico. Con base en los parámetros (media) de las funciones de distribución calculadas con el modelo bayesiano y el modelo clásico tenemos los siguientes resultados. 


\begin{tabular}{c|c|c|c}
\hline Modelo & $\begin{array}{c}\text { Frecuencia } \\
\text { promedio }(\mathbf{F})\end{array}$ & $\begin{array}{c}\text { Severidad } \\
\text { promedio }(\mathbf{S})\end{array}$ & $\begin{array}{c}\text { Pérdida promedio } \\
\text { por evento }(\mathbf{S} / \mathbf{F})\end{array}$ \\
\hline Bayesiano & 685 & 9671 & 14.11 \\
\hline Clásico & 477 & $\operatorname{Exp}(7.58)=1958$ & 4.1 \\
\hline
\end{tabular}

La pérdida promedio por evento obtenida con el modelo bayesiano es de 14 pesos, lo que se aproxima más al costo de reexpedición de un cheque cuando no se puede realizar la trasferencia electrónica de recursos a los beneficiarios; en este sentido, el resultado es consistente.

Los factores de riesgo relacionados con los procesos cuenta cancelada, cuenta en otra divisa y cuenta inexistente se traducen en un mal desempeño del proceso con probabilidad del 64\% (ver gráfica 8), lo que genera pérdidas quincenales del orden de 8554 pesos quincenales (ver gráfica 9); para mitigar estos riesgos se proponen las siguientes acciones:

- Mantener actualizado el padrón de beneficiarios.

- Evitar en la medida de lo posible pagos con ambos medios (cheque y depósito).

- Evitar la cancelación de cuentas de manera sistemática.

- Sólo trabajar con cuentas abiertas por Sagarpa.

- Incorporar la orden de pago como otro medio alternativo.

- Concluir con el proceso de bancarización.

\section{Conclusiones}

El cambio de pago por cheque a transferencia electrónica en Procampo incrementó de manera considerable la transparencia y oportunidad de la entrega de los apoyos a los beneficiarios del programa, además de reducir los costos administrativos y operativos. Sin embargo, como quedó demostrado en el desarrollo de este trabajo, existen factores de riesgo asociados al proceso de dispersión de recursos que impiden la entrega de éstos a través del depósito en la cuenta de los beneficiarios, lo que obliga a realizar el pago a través de cheques, generándose un costo adicional por cada transacción no exitosa. Los factores de riesgo que más impactan están relacionados con actividades internas para el pago de apoyos, los cuales se pueden mitigar con mejoras a los procesos de dispersión y actualización del padrón, además de continuar con la política de bancarización. 
En congruencia con lo anterior, este trabajo proporciona los elementos teóricos necesarios, así como una guía práctica para identificar, medir, cuantificar y administrar el RO en el proceso de dispersión de recursos (transferencia electrónica) con un enfoque bayesiano que mostró utilizar elementos más apegados a la realidad como probabilidades obtenidas de los expertos o externos cuando no existe información histórica, distribuciones de probabilidad específicas para cada factor de riesgo que pueden ser discretas o continuas, actualización de datos que se incorporan al modelo y la interrelación (causalidad) de los factores de riesgo a través de modelos de redes. Asimismo, se mostró que las redes bayesianas son una opción viable para administrar el riesgo operacional en un ambiente de incertidumbre y de información escasa o de calidad cuestionable. El capital requerido en riesgo operacional calculado se basa en el supuesto de interrelación (causa-efecto) entre factores de riesgo, lo cual es consistente con la realidad.

Las redes bayesianas están basadas en algoritmos eficientes de propagación de evidencias, que actualizan dinámicamente el modelo con datos actuales. Para el caso del objeto de estudio de este trabajo fue posible construir la RB y calcular el capital requerido para administrar el riesgo operacional combinando datos estadísticos, así como opiniones o juicios de los expertos o información externa.

El VaR calculado con el enfoque bayesiano es consistente en el sentido de Artzner (1998), pero también resume las complejas relaciones causales entre los diferentes factores de riesgo que derivan en un evento de riesgo operacional. En resumen, debido a que la realidad es mucho más compleja que eventos independientes idénticamente distribuidos, el enfoque bayesiano es una alternativa para modelar una realidad compleja y dinámica.

\section{Referencias}

Aquaro, V., M. Bardoscia, R. Belloti, A. Consiglio, F. De Carlo y G. Ferri (2010). A Bayesian Networks Approach to Operacional Risk. Physica A: Statistical Mechanics and its Applications 389 (8): 1721-1728.

Alexander, C. (2002). Operational Risk Measurement: Advanced Approaches. Disponible en: http://www.dofin.ase.ro/Lectures/Alexander\%20Carol/ Oprisk_Bucharest.pdf 
Artzner, P., F. Delbaen, J. Eber y D. Heath (1999). Coherent Measures of Risk. Mathematical Finance 9 (3): 203-228.

Basilea II (2001). Consultative document. Operational Risk.

Basilea II (2001). Working Paper on the Regulatory Treatment of Operational Risk.

Cowell, R. (1999). Introduction to inference for bayesian networks. In Jordan: 9-26.

Cruz, M., ed. (2004). Operational Risk Modelling and Analysis. Theory and Practice. London: Risk Books.

Degen, M., P. Embrechts y D. Lambrigger (2007). The Quantitative Modeling of Operational Risk: Between g-and-h and EVT. Disponible en: http://www. actuaires.org/ASTIN/Colloquia/Orlando/Papers/Degen.pdf

Embrechts, P., H. Furrer y O. Kaufmann (2003). Quantifying Regulatory Capital for Operational Risk. Derivatives Use, Trading and Regulation 9 (3): 217233.

Ferguson, T. S. (1973) A Bayesian Analysis of Some Nonparametric Problems. Annals of Statistics 2 (4): 615-629.

Guo, H. and W. Hsu (2002). A Survey of Algorithms for Real-Time Bayesian Network Inference. Disponible en: http://aaaipress.org/Papers/Workshops/2002/WS-02-15/WS02-15-001.pdf

Heinrich, G. (2006). Riesgo operacional, pagos, sistemas de pago y aplicación de Basilea II en América Latina: evolución más reciente. Boletín del CEMLA, octubre-diciembre: 191-204.

Ishikawa, K. (1943). Diagrama Causa-Efecto. Disponible en: http://es.wikipedia. org/wiki/Diagrama_de_Ishikawa.

Jensen, F. V.(1996). An Introduction to Bayesian Network. London: Taylor \& Francis Group. 
King, J. L. (2001). Operational Risk: Measurement and Modeling. West Sussex: John Wiley \& Sons.

Kartik, A. y K. Reimer (2003). Phase transitions in operational risk, Physical Review E, 75 (1): 016111-1-016111-12.

Lauritzen, S.L. y D.J. Spiegelhalter (1988). Local computations with probabilities on graphical structures and their applications to expert systems. In Proceedings of the Royal Statistical Society 50: 154-227.

Leippold, M. y P. Vanini (2003). The Quantification of Operational Risk. Disponible en: http://papers.ssrn.com/sol3/papers.cfm?abstract_id=481742

Moscadelli, M.(2004). The Modelling of Operational Risk: Experience with the Analysis of the Data Collected by the Basel Committee. Disponible en: http://papers.ssrn.com/sol3/papers.cfm?abstract_id=557214

Neil, M., N. Fenton y M. Tailor (2004). Using Bayesian Networks to Model Expected and Unexpected Operational Losses. Risk Analysis 25 (4): 963-972.

Panjer, H. (2006). Operational Risk Modeling Analytics. New Jersey: John Wiley $\&$ Sons.

Pearl, J. (2000). Causality, Models, Reasoning, and Inference. Cambridge: Cambridge University Press.

Reimer, K. y P. Neu (2002). Adequate Capital and Stress Testing for Operational Risks. Disponible en: http://www.mth.kcl.ac.uk/ kuehn/published/12Kuhn.pdf

(2003). Functional Correlation Approach to Operational Risk in Banking Organizations. Disponible en: http://arxiv.org/pdf/cond-mat/0204368. pdf

Svetlozar, T., J. Hsu, S. Biliana y F. Fabossi (2008). Bayesian Methods in Finance, New Jersey: Jonh Wiley \& Sons. 
Supatgiat, C., C. Kenyon y L. Heusler (2006). Cause-to-Effect Operational Risk Quantification and Management. Risk Management 8: 16-42.

Ramamurthy, S., H. Arora y A. Ghosh (2005). Operational Risk and Probabilistic Net-works an Application to Corporate Actions Processing. Disponible en: http://www.hugin.com/cases/Finance/Infosys/oprisk.article

Venegas-Martínez, F. (2006). Riesgos financieros y económicos (productos derivados y decisiones económicas bajo incertidumbre). México: International Thomson Editors.

Zellner, A. (1971). An Introduction to Bayesian Inference in Econometrics, New Jersey: John Wiley \& Sons.<smiles></smiles> 


\section{Apéndice A. Algoritmo exacto para inferencia bayesiana}

Dentro de los algoritmos de inferencia exacta tenemos el polytree (Pearl, 2000), el clique tree (Lauritzen y Spiegelhalter, 1988) y junction tree (Cowell, 1999). El método de Pearl es uno de los primeros y más utilizados. La propagación de las creencias, de acuerdo con Pearl (2000), siguen el siguiente proceso: sea $e$ el conjunto de valores para todas las variables observadas. Para cualquier variable $X$, $e$ puede dividirse en dos subconjuntos: $e_{X}^{-}$, el cual representa todas las variables observadas que descienden de $X$ y $e_{X}^{+}$, que representa todas las demás variables observadas. El impacto de las variables observadas sobre las creencias de $X$ pueden representarse a través de los siguientes dos valores:

$$
\begin{aligned}
& \lambda(X)=P\left(e_{X}^{-} \mid X\right) \\
& \pi(X)=P\left(e_{X}^{+} \mid X\right) ;
\end{aligned}
$$

$\lambda(X)$ y $\pi(X)$ son vectores cuyos elementos están asociados a cada valor de $X$ :

$$
\begin{gathered}
\lambda(X)=\left[\begin{array}{llllll}
\lambda & X=x_{1}, \lambda & X=x_{2} & , \ldots, \lambda & X=x_{l}
\end{array}\right] \\
\pi(X)=\left[\begin{array}{llllll}
\lambda & X=x_{1}, \lambda & X=x_{2} & , \ldots, \lambda & X=x_{l}
\end{array}\right]
\end{gathered}
$$

La distribución a posteriori se obtiene usando (A1) y (A2).

$$
P(X \mid e)=\alpha \lambda(X) \pi(X)
$$

donde $\alpha=1 / P(e)$ y la multiplicación por pares de los elementos en $\lambda(X)$ y $\pi(X)$. Por otro lado, para encontrar las nuevas creencias, se calcula la ecuación (A5). Los valores de $\lambda(X)$ y $\pi(X)$ se pasan entre las variables de una manera ordenada. $\lambda(X)$ y $\pi(X)$ se calculan de la siguiente manera: $\lambda(X)$ se calcula utilizando $\lambda\left(Y_{1}, Y_{2}, \ldots, Y_{m}\right)$, donde $\lambda\left(Y_{1}, Y_{2}, \ldots, Y_{m}\right)$ son hijos de $X$. Primero, cuando $X$ toma el valor $x^{0} \operatorname{los}$ elementos del vector $\lambda(X)$ se asignan de la siguiente manera:

$$
\lambda\left(x_{i}\right)= \begin{cases}0 & \text { si } x_{i} \neq x^{0} \\ 1 & \text { si } x_{i}=x^{0}\end{cases}
$$

Para el caso donde $X$ no tiene valor, tenemos $e_{X}^{-}=\bigcup_{i=1}^{m} e_{y_{i}}^{-}$. Al Utilizar (A1), $\lambda(X)$ se expande como: 


$$
\begin{gathered}
\lambda(X)=P\left(e_{X}^{-} \mid X\right) \\
=P\left(e_{y_{1}}^{-}, e_{y_{2}}^{-}, \ldots, e_{y_{m}}^{-} \mid X\right) \\
=P\left(e_{y_{1}}^{-} \mid X\right) P\left(e_{y_{2}}^{-} \mid X\right) \cdots P\left(e_{y_{m}}^{-} \mid X\right) \\
=\lambda_{y_{1}}(X) \lambda_{y_{2}}(X) \cdots \lambda_{y_{m}}(X)
\end{gathered}
$$

Usando el hecho de $e_{y_{1}}^{-}, e_{y_{2}}^{-}, \ldots, e_{y_{m}}^{-}$que son condicionalmente independientes, y definiendo lo siguiente:

$$
\lambda_{y_{i}}(X)=P\left(e_{X}^{-} \mid X\right)
$$

Se tiene que para cada $\lambda_{y_{i}}(X)$, el cálculo se realiza de la siguiente manera:

$$
\begin{aligned}
& \lambda_{y_{i}}(X)=P\left(e_{Y}^{-} \mid X\right) \\
= & \sum_{y_{i}} P\left(e_{y_{i}}^{-}, y_{i} \mid X\right) \\
= & \sum_{y_{i}} P\left(e_{y_{i}}^{-} \mid y_{i}, X\right) P\left(y_{i} \mid X\right) \\
= & \sum_{y_{i}} P\left(e_{y_{i}}^{-} \mid y_{i}\right) P\left(y_{i} \mid X\right) \\
= & \sum_{y_{i}} \lambda_{y_{i}} P\left(y_{i} \mid X\right)
\end{aligned}
$$

La expresión final muestra que para calcular el valor de $\lambda(X)$, únicamente se requieren las $\lambda$ y las probabilidades condicionales de todos los hijos de $X$. En forma compacta, el vector $\lambda(X)$ se calcula como:

$$
\lambda(X)=\Pi_{c \in \text { children }(X)} \sum_{v \in c} \lambda(v) P(v \mid X) .
$$

Para el cálculo de $\pi(X)$ se utiliza el padre $Y$ de las $X$. En efecto, usando (A2):

$$
\begin{aligned}
& \pi(X)=P\left(X \mid e_{X}^{+}\right) \\
= & \sum_{y_{i}} P\left(X, y_{i} \mid e_{X_{i}}^{+}\right)
\end{aligned}
$$




$$
\begin{aligned}
& =\sum_{y_{i}} P\left(X \mid y_{i}, e_{X_{i}}^{+}\right) P\left(y_{i} \mid e_{X}^{+}\right) \\
& =\sum_{y_{i}} P\left(X \mid y_{i}\right) P\left(y_{i} \mid e_{X}^{+}\right) \\
& =\sum_{y_{i}} P\left(X \mid y_{i}, e_{X_{i}}^{+}\right) \pi\left(y_{i}\right)
\end{aligned}
$$

Lo anterior muestra que para calcular $\pi(X)$, se requieren las $\pi$ de los padres $X$, así como sus probabilidades condicionales.

Se pueden presentar problemas con el método de inferencia de Pearl debido a los ciclos que se generan cuando se elimina la direccionalidad (ver Pearl, 1988, capítulo 44). Por tanto, el algoritmo junction tree de Cowell (1999) ha resultado más útil y práctico: convierte la gráfica dirigida en un árbol cuyos nodos son cerrados para proceder a propagar los valores de $\lambda$ y $\pi$ a través del árbol; el proceso es el siguiente:

1. Moralizar la red bayesiana, esto significa relacionar por pares a los padres de cada nodo.

2. Triangular la gráfica anterior.

3. Identificar los nodos máximos y completos para construir el árbol, el cual se convertirá en el junction tree.

4. Propagar los valores de $\lambda$ y $\pi$ a través del árbol para generar la inferencia, o probabilidades a posteriori. 
